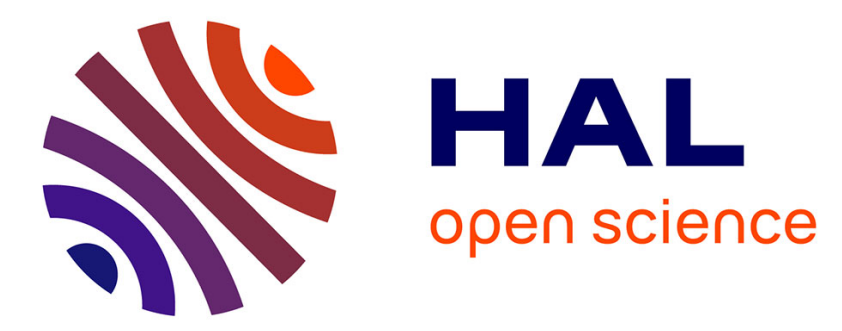

\title{
Approximate maximum likelihood estimation of two closely spaced sources
}

François Vincent, Olivier Besson, Eric Chaumette

\section{To cite this version:}

François Vincent, Olivier Besson, Eric Chaumette. Approximate maximum likelihood estimation of two closely spaced sources. Signal Processing, 2014, vol. 97, pp. 83-90. 10.1016/j.sigpro.2013.10.017 . hal-00905319

\section{HAL Id: hal-00905319 https://hal.science/hal-00905319}

Submitted on 18 Nov 2013

HAL is a multi-disciplinary open access archive for the deposit and dissemination of scientific research documents, whether they are published or not. The documents may come from teaching and research institutions in France or abroad, or from public or private research centers.
L'archive ouverte pluridisciplinaire HAL, est destinée au dépôt et à la diffusion de documents scientifiques de niveau recherche, publiés ou non, émanant des établissements d'enseignement et de recherche français ou étrangers, des laboratoires publics ou privés. 


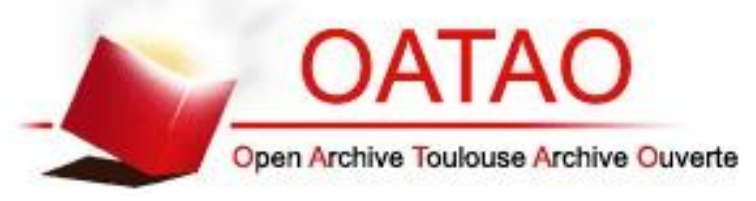

\section{Open Archive Toulouse Archive Ouverte (OATAO)}

OATAO is an open access repository that collects the work of Toulouse researchers and makes it freely available over the web where possible.

This is an author-deposited version published in: http://oatao.univ-toulouse.fr/ Eprints ID: 10106

To link to this article: DOI: 10.1016/j.sigpro.2013.10.017

URL: http://dx.doi.org/10.1016/j.sigpro.2013.10.017

To cite this version: Vincent, François and Besson, Olivier and Chaumette, Eric Approximate maximum likelihood estimation of two closely spaced sources. (2014) Signal Processing, vol. 97. pp. 83-90. ISSN 0165-1684 


\title{
Approximate maximum likelihood estimation of two closely spaced sources
}

\author{
François Vincent $^{\mathrm{a}, *}$, Olivier Besson ${ }^{\mathrm{a}}$, Eric Chaumette ${ }^{\mathrm{b}}$ \\ ${ }^{a}$ University of Toulouse-ISAE, Department of Electronics, Optronics and Signal, 10 Avenue Edouard Belin, 31055 Toulouse, France \\ ${ }^{\mathrm{b}}$ ONERA, The French Aerospace Lab, DEMR/TSI, 91120 Palaiseau, France
}

A R T I C L E I N F O

Keywords:

Approximate maximum likelihood

estimation

Closely spaced sources

Direction finding

\begin{abstract}
A B S T R A C T
The performance of the majority of high resolution algorithms designed for either spectral analysis or Direction-of-Arrival (DoA) estimation drastically degrades when the amplitude sources are highly correlated or when the number of available snapshots is very small and possibly less than the number of sources. Under such circumstances, only Maximum Likelihood (ML) or ML-based techniques can still be effective. The main drawback of such optimal solutions lies in their high computational load. In this paper we propose a computationally efficient approximate ML estimator, in the case of two closely spaced signals, that can be used even in the single snapshot case. Our approach relies on Taylor series expansion of the projection onto the signal subspace and can be implemented through 1D Fourier transforms. Its effectiveness is illustrated in complicated scenarios with very low sample support and possibly correlated sources, where it is shown to outperform conventional estimators.
\end{abstract}

\section{Introduction}

Identifying multiple superimposed exponential signals in noise is a central problem in many signal processing applications such as radar, sonar, telecommunications, seismology, medicine, radio-astronomy [1]. This problem has received considerable attention during the last 40 years, both for time series analysis and array processing. In the first case, one usually has to estimate the frequencies of complex sine waves from a single experiment data. In the second case, one looks for the directions of arrival (or spatial frequencies) of multiple plane waves impinging on a narrow-band array of sensors using multiple snapshots. Among the huge variety of solutions, ML was one of the first to be investigated [2] because of its well grounded principles and its optimal statistical properties, at least

\footnotetext{
* Corresponding author. Tel.: +33 561339247.

E-mail addresses: francois.vincent@isae.fr (F. Vincent), olivier.besson@isae.fr (O. Besson)

eric.chaumette@onera.fr (E. Chaumette).
}

asymptotically [3]. Two kinds of ML solutions have been derived depending on the statistical assumptions about the signals impinging on the array, namely the conditional ML estimator (CMLE) which considers the sources amplitude as unknown deterministic variables [4] and the unconditional ML estimator (UMLE) which assumes the sources amplitude to be independent and identically distributed random variables usually drawn from Gaussian distribution. The corresponding Cramer-Rao Bound (CRB) has been derived for each model [4] and it has been shown that UMLE is efficient whereas CMLE is not, mainly because the number of unknown parameters grows with the number of snapshots. However, when the signal to noise ratio (SNR) increases, the covariance matrix of both UMLE and CMLE tends to the same asymptotic CRB [4]. In this paper, we adopt the less constrained CMLE framework: indeed, assuming that the sources are temporally white Gaussian process which is a strong hypothesis that could fail in many real-life applications. Despite their nearly optimal properties (in the asymptotic regime), ML estimators suffer from a large computational cost, as they require solving 
a non-linear multidimensional optimization problem. Hence, the signal processing community has focused on two different ways to circumvent this problem.

A first approach consists in using sub-optimal techniques to reduce computational burden. Many methods substitute the multidimensional search for a simpler one-dimensional search, see e.g., the well-known Capon [5] or MUSIC [6,7] methods. In the case of a uniform linear array (ULA), other techniques directly provide the estimates from a polynomial root search, see e.g., root-MUSIC [8,9], ESPRIT [10], MinNorm [11] and modified versions thereof such as unitary root-Music [12] or unitary-ESPRIT [13]. All these techniques could achieve near-ML performance in many cases [14] and, for instance, MUSIC can be interpreted as a large sample realization of the MLE if the sources are uncorrelated [15]. The two main drawbacks of subspace-based methods concern the case where the sources are highly correlated or coherent (multipath propagation for instance) or when the number of available snapshots is less than the number of sources. In the former case, some techniques such as spatial smoothing [16] have proven to be efficient in mitigating sources correlation, but at the price of a loss of resolution, which is problematic in the case of closely spaced signals where high-resolution is needed. The sample starving scenario occurs with highly non-stationary environments such as embedded radars (in automotive, jetfighter or missile) where the stationary duration is very small. In the case of automotive radars, asymptotic performance of DoA estimation has been analyzed in [17] and $[18,19]$ provide a good overview of techniques that can practically be used in this case. Whenever the number of snapshots becomes less than the number of sources, subspace-based methods are no longer appropriate since the signal subspace cannot be retrieved. However, ML techniques are still able to handle this type of situation.

Consequently, a second class of methods aims at exploiting the ML principle but with a reduced computational burden. Their performance can then come close to that of the MLE even in the case of coherent sources or single snapshot data-set. The main idea behind all these techniques is to split the multidimensional optimization procedure in a number of recursive and simpler searches. Two kinds of computational cost reduction have been used in the literature. The first type of method is based on relaxation procedures and lead to the Alternating Projection algorithm when apply to CMLE and to the Alternating Maximization procedure when apply to UMLE [20]. The second kind relies on the Expectation Maximization principle [21] and has been used in the array processing context by Weiss [22] for instance. In the case of a ULA, exploiting the equivalence between the noise subspace and the prediction polynomial coefficients subspace, Bresler has developed the so-called IQML algorithm [23] and Stoica the so-called IMODE algorithm [24] that are both recursive simplified procedures to minimize the CMLE criterion. Nevertheless, these simplified implementations of the MLE require a good initialization point and are not guaranteed to converge to the global minimum. Moreover, the recursive nature of these procedures is hardly compatible with covariance matrix update algorithms optimized for tracking modes [25].
In this paper, we propose a non-recursive, one-dimensional, low cost minimization procedure to estimate the frequencies of two closely spaced signals. The computational cost of this new procedure is in the same order of classical Fourier transforms. Additionally, this solution is a close approximation of the CMLE and inherits its good properties, namely robustness to coherent sources and to low number of snapshots. Moreover, it is shown to be more accurate than conventional root-MUSIC or ESPRIT estimators even in the case of uncorrelated sources. At last, it can also be used in the case of a single snapshot where high resolution techniques are very scarce despite the widespread interest of such a scenario. The present procedure is developed in the case of two sources, a natural framework when dealing with high resolution [26-28]. Indeed, in a large number of real-life signal processing applications, the number of sources to identify is known to be two or less, because matched preprocessing techniques have reduced this number. For instance, in radar or sonar processing, DOA estimation is performed after delay and frequency filtering so that one just focuses on a single range-Doppler bin. The joint problem of detection of the number of sources is out of the scope of this paper, but we would like to point out that in some practical situations, one a priori knows that two sources exactly are present. This is the case for instance when a radar is looking for a low elevation target over the sea [29]. The received signal is systematically composed of a direct path and a close angle multipath. Except MLE, high-resolution procedures cannot be used in this situation because of a strong correlation between the two signals, and the approach proposed herein is relevant.

The paper is organized as follows. Section 2 introduces the framework at hand and gives a brief reminder on CMLE and the associated CRB. Then, in Section 3, a Taylor series expansion of the signal subspace projector with respect to the frequency difference between sources is carried out, which results in a closed-form expression of the ML estimate. When plugged back in the likelihood function, we end up with a simple one-dimensional search. Numerical simulations illustrate the superiority of this estimate compared with classical subspace-based techniques in Section 4, and our conclusions are drawn in Section 5.

\section{Data model}

We consider a narrow-band uniform linear array of $M$ sensors with inter-element spacing $d$. We assume that two plane waves impinge on the array with respective DoA $\theta_{1}$ and $\theta_{2}$ and we consider a scenario where the two DoA are very close, typically within a fraction of the array beam width. Let $f_{i}=(d / \lambda) \sin \theta_{i}, i=1,2$ denote the corresponding spatial frequencies and let us re-parameterize the problem in terms of $f_{1}$ and $\Delta_{f}=f_{2}-f_{1}$, where, by assumption, $\Delta_{f} \ll 1 / M$. The model at hand can then be written as follows:

$\boldsymbol{x}_{t}=\boldsymbol{A}\left(f_{1}, \Delta_{f}\right) \boldsymbol{s}_{t}+\boldsymbol{n}_{t}, \quad t=0, \ldots,(N-1)$ 
where

- $\boldsymbol{A}\left(f_{1}, \Delta_{f}\right)=\left[\boldsymbol{a}\left(f_{1}\right) \boldsymbol{a}\left(f_{1}+\Delta_{f}\right)\right] \in \mathbb{C}^{M \times 2}$ with $\boldsymbol{a}(f)=\frac{1}{\sqrt{M}}\left[\begin{array}{llll}1 & e^{2 i \pi f} & \cdots & e^{2 i \pi f(M-1)}\end{array}\right]^{T}$ denoting the normalized steering vector

- $\boldsymbol{s}_{t} \in \mathbb{C}^{2}$ stands for the vector of unknown deterministic amplitudes of the sources, similar to the CMLE framework

- $\boldsymbol{n}_{t} \in \mathbb{C}^{M}$ denotes the noise vector and is assumed to be zero-mean circularly Gaussian with covariance matrix $\sigma^{2} \boldsymbol{I}$ where $\sigma^{2}$ is an unknown scalar. Moreover, $\boldsymbol{n}_{t}$ is supposed to be temporally white, so that $\mathcal{E}\left\{\boldsymbol{n}_{t} \boldsymbol{n}_{s}^{H}\right\}=$ $\sigma^{2} \mathbf{I} \delta_{t-s}$ and $\mathcal{E}\left\{\boldsymbol{n}_{t} \boldsymbol{n}_{s}^{T}\right\}=\mathbf{0} . \delta$ stands for the Kronecker symbol $\left(\delta_{0}=1\right.$ and $\delta_{t-s}=0$ if $\left.t \neq s\right)$

It can be noticed that in the single snapshot case $(N=1)$, this model is also valid for spectral analysis in time series analysis. The problem at hand consists in estimating $f_{1}$ and the frequency difference $\Delta_{f}$. The ML solution is obtained by maximizing the log-likelihood function with respect to the unknown parameters. Concentrating the likelihood function with respect to $\sigma^{2}$ and all $\boldsymbol{s}_{t}$, it is well known that the MLE of $f_{1}$ and $\Delta_{f}$ is given by [14]

$\hat{f}_{1}, \hat{\Delta}_{f}=\arg \min _{f_{1}, \Delta_{f}} \sum_{t=0}^{N-1}\left\|\boldsymbol{x}_{t}-\boldsymbol{P}\left(f_{1}, \Delta_{f}\right) \boldsymbol{x}_{t}\right\|^{2}$

$\hat{f}_{1}, \hat{\Delta}_{f}=\arg \min _{f_{1}, \Delta_{f}} \operatorname{Tr}\left\{\boldsymbol{P}^{\perp}\left(f_{1}, \Delta_{f}\right) \hat{\boldsymbol{R}}\right\}$

$\hat{f}_{1}, \hat{\Delta}_{f}=\arg \max _{f_{1}, \Delta_{f}} \sum_{t=0}^{N-1}\left\|\boldsymbol{P}\left(f_{1}, \Delta_{f}\right) \boldsymbol{x}_{t}\right\|^{2}$

where $\operatorname{Tr}\{\}$ stands for the trace of the matrix between braces,

$\boldsymbol{P}\left(f_{1}, \Delta_{f}\right)=\boldsymbol{A}\left(f_{1}, \Delta_{f}\right)\left(\boldsymbol{A}^{H}\left(f_{1}, \Delta_{f}\right) \boldsymbol{A}\left(f_{1}, \Delta_{f}\right)\right)^{-1} \boldsymbol{A}^{H}\left(f_{1}, \Delta_{f}\right)$

is the projection onto the subspace spanned by the columns of $\boldsymbol{A}\left(f_{1}, \Delta_{f}\right)$ (signal subspace) and $\boldsymbol{P}^{\perp}\left(f_{1}, \Delta_{f}\right)=\boldsymbol{I}-\boldsymbol{P}\left(f_{1}, \Delta_{f}\right)$ is the projection onto the noise subspace. Finally

$\hat{\boldsymbol{R}}=\frac{1}{N} \sum_{t=0}^{N-1} \boldsymbol{x}_{t} \boldsymbol{x}_{t}^{H}$

is the sample covariance matrix. Eq. (2) proposes an appealing geometric interpretation of the ML estimates, viz., we are simply looking for the frequencies that generate the closest subspace to the data. Associated with this deterministic model, one can also derive the CRB as [14]

$\boldsymbol{B}_{c}=\frac{\sigma^{2}}{2 N} \operatorname{Re}\left[\hat{\boldsymbol{R}}_{s} \odot \boldsymbol{H}^{T}\right]^{-1}$

where $\odot$ stands for the Hadamard (elementwise) product $\hat{\boldsymbol{R}}_{s}=(1 / N) \sum_{t=0}^{N-1} \boldsymbol{s}_{t} \boldsymbol{s}_{t}^{H}$ is the sources amplitude covariance matrix estimate of $\boldsymbol{R}_{s}=\mathcal{E}\left\{\boldsymbol{s}_{t} \boldsymbol{s}_{t}^{H}\right\}$, and $\boldsymbol{H}=\boldsymbol{\Delta}^{H} \boldsymbol{P}^{\perp} \boldsymbol{\Delta}$ with $\boldsymbol{\Delta}=\left[\partial \boldsymbol{a}(f) /\left.\partial f\right|_{f_{1}} \partial \boldsymbol{a}(f) /\left.\partial f\right|_{f_{1}+\Delta_{f}}\right]$. Of course, this lower bound on the frequency parameters depends on $\boldsymbol{s}_{t}$ and can thus vary from one set of observed data to another. Hence, some sort of averaging is usually conducted. Alternatively, one may wish to compare the performance of any estimator to the asymptotic $(N \rightarrow \infty)$ limit of $(5)$, viz.,

$\boldsymbol{B}_{c}^{a s}=\frac{\sigma^{2}}{2 N} \operatorname{Re}\left[\boldsymbol{R}_{s} \odot \boldsymbol{H}^{T}\right]^{-1}$.

\section{Approximate maximum likelihood estimation for two closely spaced sources}

In this section, we focus on the case of two unresolved targets or close-frequency signals. In this case, we conduct a Taylor series expansion of the ML criterion with respect to the frequency difference $\Delta_{f}$. Let us start with the signal subspace projection matrix. From the definition of $\boldsymbol{A}\left(f_{1}, \Delta_{f}\right)$ we have

$\left(\boldsymbol{A}^{H}\left(f_{1}, \Delta_{f}\right) \boldsymbol{A}\left(f_{1}, \Delta_{f}\right)\right)^{-1}=\frac{1}{1-\left|c\left(\Delta_{f}\right)\right|^{2}}\left(\begin{array}{cc}1 & -c\left(\Delta_{f}\right) \\ -c\left(\Delta_{f}\right)^{*} & 1\end{array}\right)$

with $c\left(\Delta_{f}\right)=\boldsymbol{a}\left(f_{1}\right)^{H} \boldsymbol{a}\left(f_{1}+\Delta_{f}\right)$. In the case of a ULA one simply has $c\left(\Delta_{f}\right)=(1 / M) e^{i \pi(M-1) \Delta_{f}} \sin \pi M \Delta_{f} / \sin \pi \Delta_{f}$. However, we would like to stress the fact that the only hypothesis we really need for the derivations to follow is that $c$ only depends on $\Delta_{f}$. Under this hypothesis, we have that $\boldsymbol{a}\left(f_{1}+\Delta_{f}\right)=\boldsymbol{D}\left(\Delta_{f}\right) \boldsymbol{a}\left(f_{1}\right)$ where $\boldsymbol{D}\left(\Delta_{f}\right)=\operatorname{diag}\left(\left[1 e^{2 i \pi \Delta_{f}} \ldots\right.\right.$ $\left.\left.e^{2 i \pi \Delta_{f}(M-1)}\right]\right)$ in the case of ULA. Hence

$\boldsymbol{P}=\frac{1}{1-|c|^{2}}\left[\boldsymbol{a a}^{H}+\boldsymbol{D a a}^{H} \boldsymbol{D}^{H}-c \boldsymbol{a a} \boldsymbol{a}^{H} \boldsymbol{D}^{H}-c^{*} \boldsymbol{D a a}{ }^{H}\right]$

where, for the sake of notational simplicity, we have temporarily dropped the dependence with respect to $f_{1}$ or $\Delta_{f}$, i.e., $\boldsymbol{a}=\boldsymbol{a}\left(f_{1}\right), c=c\left(\Delta_{f}\right), \boldsymbol{D}=\boldsymbol{D}\left(\Delta_{f}\right)$ and $\boldsymbol{P}=\boldsymbol{P}\left(f_{1}, \Delta_{f}\right)$. As we are interested in the case where $\Delta_{f} \ll 1$, we can conduct a second order Taylor expansion of $\boldsymbol{P}$, for fixed $f_{1}$. Towards this end, let us write the Taylor expansions of both $\boldsymbol{D}$ and $c$ as

$\boldsymbol{D}=\sum \boldsymbol{D}_{k} \Delta_{f}^{k} \quad$ with $\quad \boldsymbol{D}_{k}=\frac{(2 i \pi)^{k}}{k !} \operatorname{diag}\left(\left[\begin{array}{ll}0^{k} & 1^{k} \cdots(M-1)^{k}\end{array}\right]\right)$

for ULA

$c=\sum c_{k} \Delta_{f}^{k}, \quad c_{k}=\frac{\operatorname{Tr}\left\{\boldsymbol{D}_{k}\right\}}{M}$.

Substituting these expressions into Eq. (8), we obtain, after some tedious albeit straightforward calculations (see Appendix), the following expansion for $\boldsymbol{P}$ :

$\boldsymbol{P} \simeq \frac{1}{d_{2}}\left(\boldsymbol{M}_{2}+\boldsymbol{M}_{3} \Delta_{f}+\left(\boldsymbol{M}_{4}-\frac{d_{4}}{d_{2}} \boldsymbol{M}_{2}\right) \Delta_{f}^{2}\right)$

where

$$
\begin{aligned}
& d_{2}=2 c_{2}-c_{1}^{2} \\
& d_{4}=2 c_{4}+c_{2}^{2}-2 c_{1} c_{3} \\
& \boldsymbol{M}_{2}=-\boldsymbol{D}_{1} \boldsymbol{a} \boldsymbol{a}^{H} \boldsymbol{D}_{1}+c_{1}\left(\boldsymbol{D}_{1} \boldsymbol{a a ^ { H }}+\boldsymbol{a a ^ { H }} \boldsymbol{D}_{1}\right)-2 c_{2} \boldsymbol{a a}^{H} \\
& \boldsymbol{M}_{3}=\boldsymbol{D}_{1} \boldsymbol{a} \boldsymbol{a}^{H} \boldsymbol{D}_{2}-\boldsymbol{D}_{2} \boldsymbol{a} \boldsymbol{a}^{H} \boldsymbol{D}_{1}+c_{1}\left(\boldsymbol{D}_{2} \boldsymbol{a} \boldsymbol{a}^{H}-\boldsymbol{a} \boldsymbol{a}^{H} \boldsymbol{D}_{2}\right) \\
& -c_{2}\left(\boldsymbol{D}_{1} \boldsymbol{a a ^ { H }}-\boldsymbol{a a ^ { H }} \boldsymbol{D}_{1}\right) \\
& \boldsymbol{M}_{4}=\boldsymbol{D}_{2} \boldsymbol{a} \boldsymbol{a}^{H} D_{2}-\boldsymbol{D}_{1} \boldsymbol{a} \boldsymbol{a}^{H} \boldsymbol{D}_{3}-\boldsymbol{D}_{3} \boldsymbol{a} \boldsymbol{a}^{H} \boldsymbol{D}_{1}+c_{1}\left(\boldsymbol{D}_{3} \boldsymbol{a a ^ { H }}+\boldsymbol{a} \boldsymbol{a}^{H} \boldsymbol{D}_{3}\right) \\
& -c_{2}\left(\boldsymbol{D}_{2} \boldsymbol{a a ^ { H }}+\boldsymbol{a} \boldsymbol{a}^{H} \boldsymbol{D}_{2}\right)+c_{3}\left(\boldsymbol{D}_{1} \boldsymbol{a a ^ { H }}+\boldsymbol{a a ^ { H }} \boldsymbol{D}_{1}\right)-2 c_{4} \boldsymbol{a a}^{H} .
\end{aligned}
$$


Substituting (11) into (4) and differentiating with respect to $\Delta_{f}$, the following closed-form expression of the frequency difference is obtained:

$\Delta_{f}^{A M L}\left(f_{1}\right)=\frac{\operatorname{Tr}\left\{\boldsymbol{M}_{3} \hat{\boldsymbol{R}}\right\}}{2 \operatorname{Tr}\left\{\left(\frac{d_{4}}{d_{2}} \boldsymbol{M}_{2}-\boldsymbol{M}_{4}\right) \hat{\boldsymbol{R}}\right\}}$

where we emphasize that $\Delta_{f}^{A M L}$ depends on $f_{1}$. The above equation constitutes the main result of this paper. It provides a closed-form expression of the ML estimate of the difference between the two frequencies. This expression can be exploited in different ways. We have chosen here a direct and robust way to solve the ML problem. More precisely, we search for the global maximum of (4) using the value of $\Delta_{f}$ in (12), using a 1D grid-based maximum search of the resulting function of $f_{1}$. In other words, we choose to estimate $f_{1}$ as

$\hat{f}_{1}^{A M L}=\arg \max _{f_{1}} \sum_{t=0}^{N-1}\left\|\boldsymbol{P}\left(f_{1}, \Delta_{f}^{A M L}\left(f_{1}\right)\right) \boldsymbol{x}_{t}\right\|^{2}$.

Once $\hat{f}_{1}^{A M L}$ is obtained, the estimate of $\Delta_{f}$ follows from (12) where $f_{1}$ is substituted for $\hat{f}_{1}^{A M L}$. It is noteworthy that evaluation of both (4) and (12) can be easily done using fast Fourier algorithms. Indeed, both equations are linear combination of terms of the following type:

$\operatorname{Tr}\left\{\boldsymbol{D}_{\ell}^{H} \boldsymbol{a}(f) \boldsymbol{a}^{H}(f) \boldsymbol{D}_{n} \hat{\boldsymbol{R}}\right\}$

which can be calculated as follows:

$$
\begin{aligned}
\operatorname{Tr}\left\{\boldsymbol{D}_{\ell}^{H} \boldsymbol{a}(f) \boldsymbol{a}^{H}(f) \boldsymbol{D}_{n} \hat{\boldsymbol{R}}\right\} & =\sum_{t=0}^{N-1} \boldsymbol{x}_{t}^{H} \boldsymbol{D}_{\ell}^{H} \boldsymbol{a}(f) \boldsymbol{a}^{H}(f) \boldsymbol{D}_{n} \boldsymbol{x}_{t} \\
& =\sum_{t=0}^{N-1} X_{t}^{\ell}(f)^{*} X_{t}^{n}(f)
\end{aligned}
$$

where $X_{t}^{\mathrm{n}}(f)=\boldsymbol{a}(f)^{H} \boldsymbol{D}_{n} \boldsymbol{x}_{t}$ is the Fourier Transform of the weighted version of $\boldsymbol{x}_{t}$ by the diagonal elements of $\boldsymbol{D}_{n}$. Therefore, $\Delta_{f}^{A M L}\left(f_{1}\right)$ in (12) can be computed from combinations of $X_{t}^{n}(f)$ for $n=0,1,2,3$. So we need to compute four Fourier transforms and eight dot-products of these vectors to evaluate $\Delta_{f}^{A M L}\left(f_{1}\right)$ for all $f_{1}$.

\section{Numerical illustrations}

In this section, we evaluate the performance of the above derived AML estimate and we compare it to the exact MLE based on a 2D search over $f_{1}$ and $f_{2}$, as well as to two conventional methods namely ESPRIT and rootMUSIC. The mean square error (MSE) for estimation of the vector $\left[f_{1} f_{2}\right]^{T}$ will serve as the figure of merit and it will be compared to the asymptotic CRB of Eq. (6). Three scenarios are considered:

- An "ideal" scenario where the number of snapshots is large enough $(N=3 M)$ and where the sources are uncorrelated $\left(\boldsymbol{R}_{s}=\boldsymbol{I}\right)$. This scenario is aimed at comparing the AMLE to subspace-based methods when the latter are likely to perform very well.

- A scenario where the sources are correlated i.e., $\boldsymbol{R}_{s}=$ $\left(\begin{array}{cc}1 & \rho \\ \rho^{*} & 1\end{array}\right)$ yet with a large number of snapshots $(N=3 M)$.
In such a case, we study the sensitivity of the AMLE to source correlation, knowing that the latter is usually detrimental to subspace-based methods.

- The single-snapshot scenario $(N=1)$, where, unless a preprocessing scheme such as spatial smoothing is used, classical subspace-based methods cannot be implemented while MLE is feasible.

In all simulations we consider a uniformly spaced linear array of $M=8$ isotropic sensors. The spatial frequencies of the sources are $f_{1}=0.1$ and $f_{1}+\Delta_{f}$ with $\Delta_{f}=1 / 10 M$ $\left(\Delta_{f}=1 / 5 M\right.$ in the single snapshot case). The MSE is computed from 1000 Monte-Carlo runs where the Gaussian vectors $\boldsymbol{n}_{t}$ and $\boldsymbol{s}_{t}$ vary in each trial. The signal to noise ratio (SNR) is defined as

$$
S N R=\frac{\operatorname{Tr}\left\{\boldsymbol{A} \boldsymbol{R}_{S} \boldsymbol{A}^{H}\right\}}{M \sigma^{2}} \text {. }
$$

\subsection{Large sample scenario, uncorrelated sources}

In this first scenario, all conditions are met to have an optimal behavior for all DOA estimation procedures. In Fig. 1, the performance comparison is displayed as a function of SNR in order to identify the so-called threshold region where the MSE departs from the CRB. We can first notice that all four methods attain the asymptotic CRB in the asymptotic region (high SNR). More interesting is the threshold region where we can notice that both the exact MLE and AMLE have a similar behavior and depart from the CRB about 2-3 dB after ESPRIT, and the latter achieves a 2-3 dB gain compared to root-MUSIC. Therefore, the proposed AML estimator has a performance very close to that of the exact MLE in the case of very closely spaced sources, and performs better than ESPRIT and root-MUSIC in this simple scenario. We now investigate its performance as a function of $\Delta_{f}$ : indeed, the Taylor series expansion is valid only for very small $\Delta_{f}$ and we wish to investigate its validity whenever $\Delta_{f}$ increases. This is done in Fig. 2 where we show the MSE of the AML for a frequency difference increasing from zero to half the main lobe width. It can be observed that the AML estimator remains close to the MLE until source separation reaches approximately a third of the beam width. We can also point out that the divergence of the AMLE from this point is quite progressive, so that the MSE keeps within a $15 \mathrm{~dB}$ range from the CRB even if the sources separation increases to half the beam width. However, for such large DoA separation high resolution methods are not really needed. Therefore, we can conclude that the proposed procedure is nearly efficient (for a small source separation) in this ideal scenario. We can even notice a lightly better behavior of the AMLE in the threshold region that suggests a kind of robustness of the proposed method.

\subsection{Large sample scenario, correlated sources}

We now examine the robustness of the AMLE towards correlation among the two sources. Fig. 3 displays the MSE of the four previous algorithms for a correlation coefficient 
Mean Square Error of Frequencies Estimates

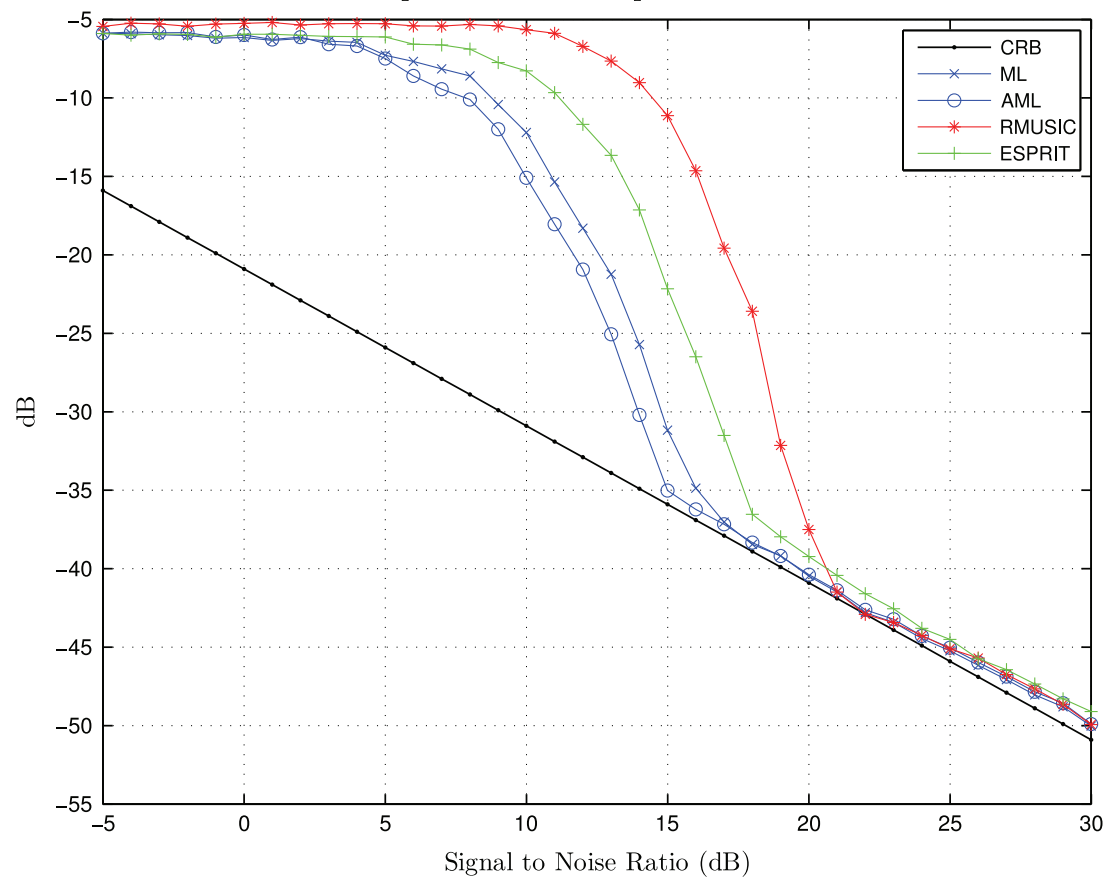

Fig. 1. Large sample scenario, uncorrelated sources. MSE of the frequency estimates versus SNR. $M=8, N=3 M, f_{1}=0.1$ and $\Delta_{f}=1 / 10 M$.

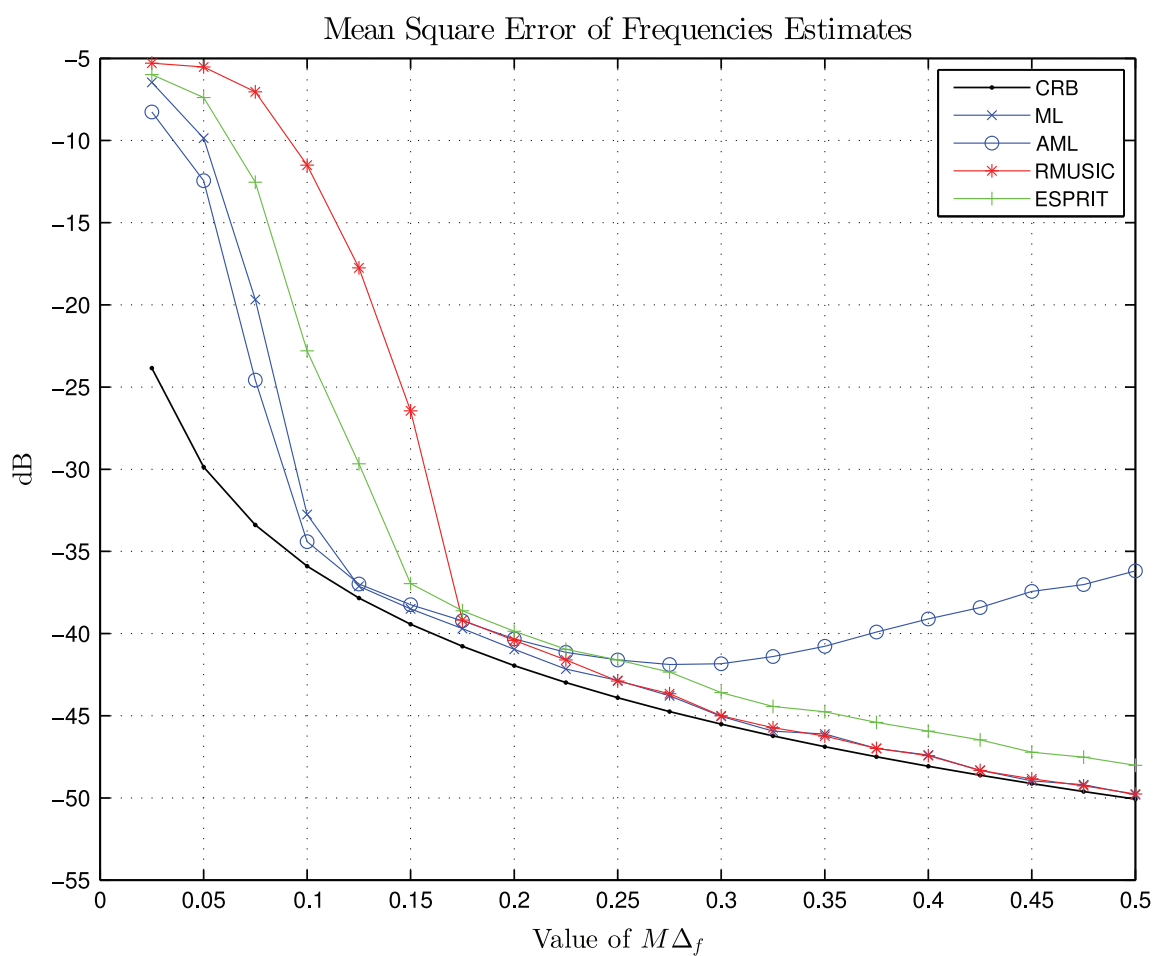

Fig. 2. Large sample scenario, uncorrelated sources. MSE of the frequency estimates versus $M \Delta_{f} . M=8, N=3 M, f_{1}=0.1$ and $S N R=15 \mathrm{~dB}$.

$\rho$ varying from 0 (previous case) to 1 (coherent sources). The input SNR is $20 \mathrm{~dB}$, for which all algorithms achieve the CRB in the case of non-correlated sources. We can see that root-MUSIC departs from the CRB even for small correlation coefficient values. ESPRIT is more robust to correlation as its divergence is more progressive. Both MLE and AMLE are more robust and remain very close to the CRB up to $\rho=0.5$. 


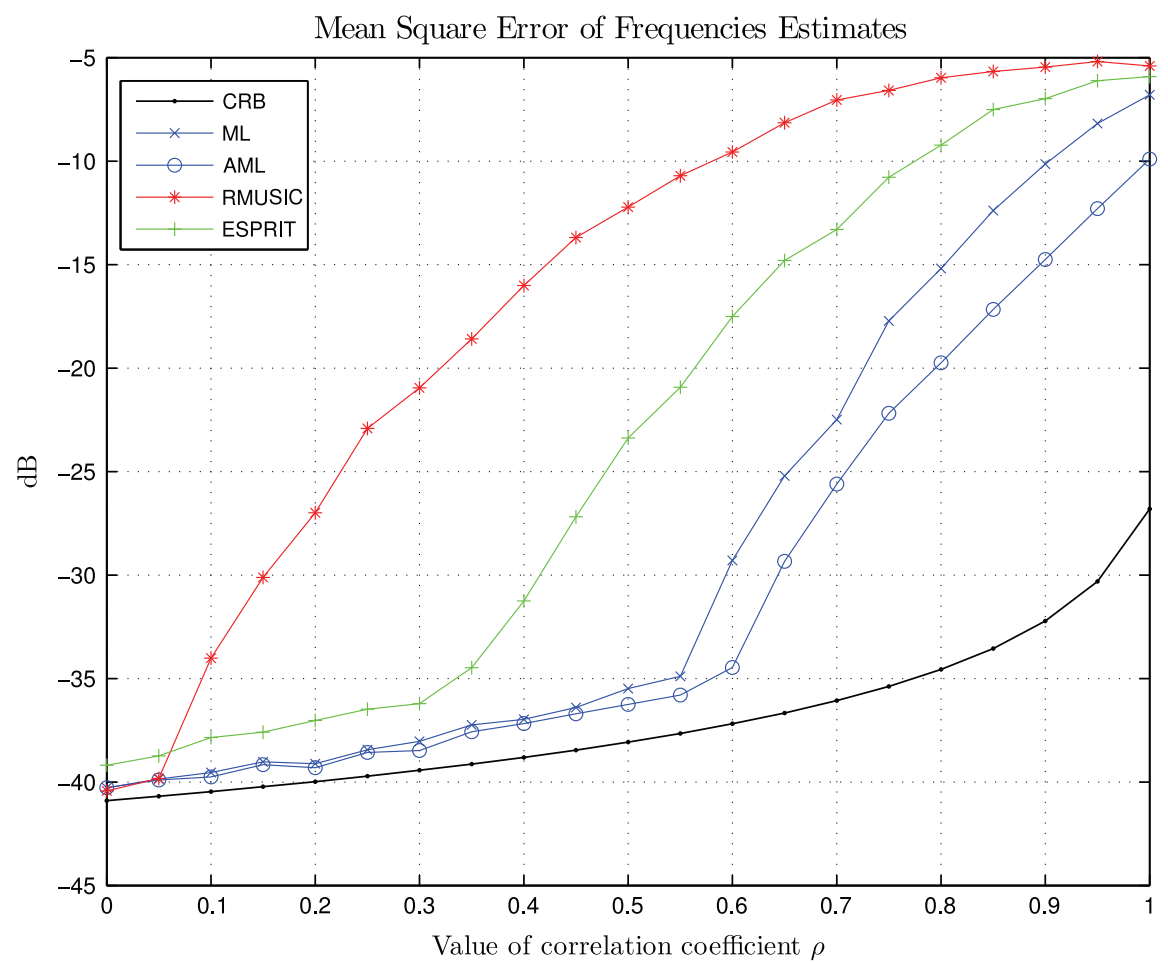

Fig. 3. Large sample scenario, correlated sources. MSE of the frequency estimates versus correlation coefficient $\rho . M=8, N=3 M, f_{1}=0.1, \Delta_{f}=1 / 10 M$ and $S N R=20 \mathrm{~dB}$.

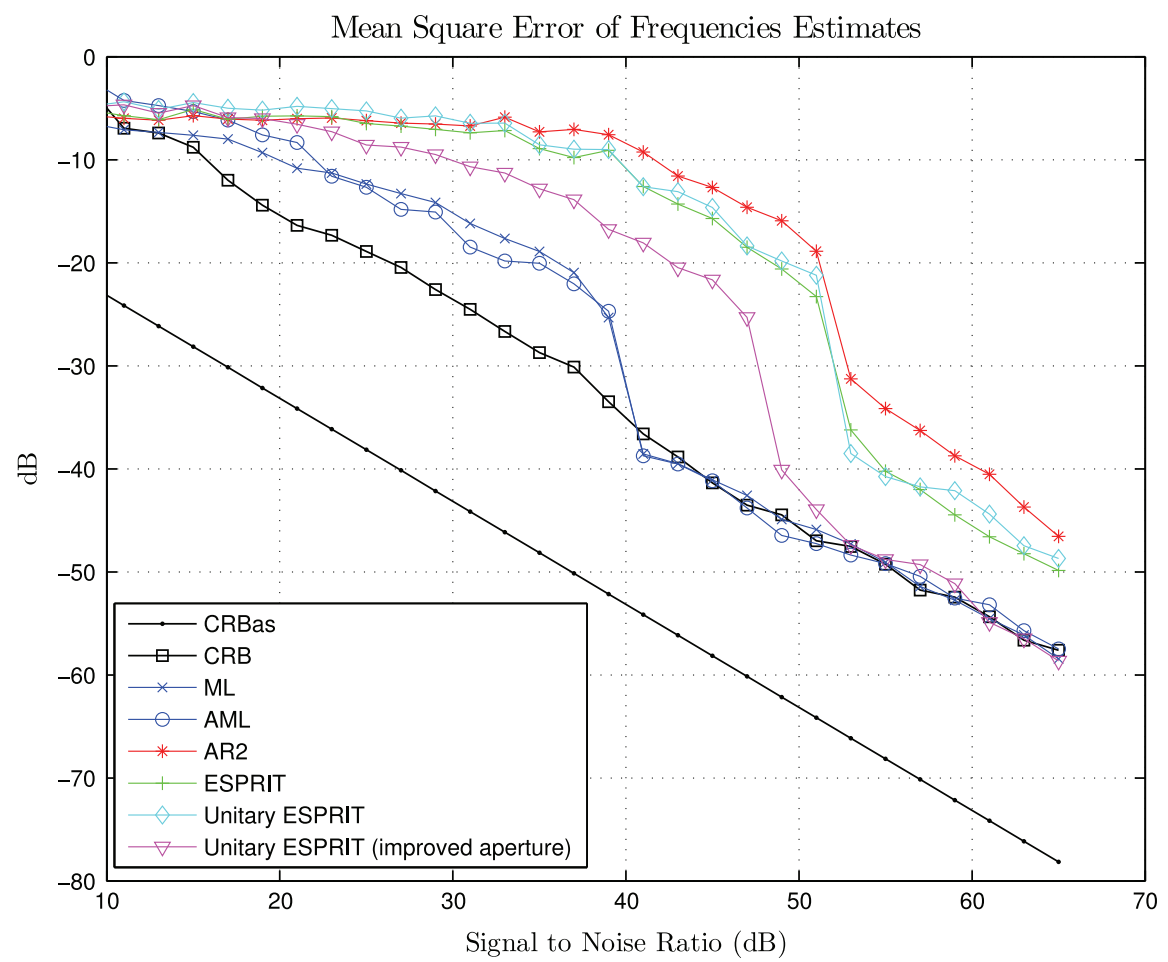

Fig. 4. The single snapshot scenario, uncorrelated sources. MSE of the frequency estimates versus SNR. $M=8, f_{1}=0.1$ and $\Delta_{f}=1 / 5 M$. 


\subsection{The single snapshot scenario}

In the single snapshot scenario, neither ESPRIT nor root-MUSIC can be used directly since a rank-two subspace cannot be retrieved from one snapshot. In fact, very few direct high resolution methods can be employed in this case. In order for subspace-based methods to be used, one has to substitute missing snapshots for real sensor data as done e.g., in spatial smoothing techniques. The price to be paid will be a reduction of the array aperture and consequently a loss of resolution. The effects of such a procedure will be all the more detrimental that the number of sensors is small and the number of sources is high. In our case of interest, we have chosen to compare AMLE and MLE to a spectral estimation based on an AR(2) modeling. The two AR coefficients are computed from a least-squares algorithm and the two frequencies of interest from a polynomial root search. Moreover, we also provide a comparison with ESPRIT, Unitary ESPRIT and Unitary ESPRIT with improved aperture [30] procedures. In the latter cases, we perform averaging over three subarrays, each one comprising six elements. Fig. 4 compares the performances of these estimators. The average value of the exact CRB of (5) is also plotted. We can first see that in this single snapshot case, no considered methods achieve the asymptotic CRB, but that both MLE and AMLE are very close to the exact CRB (nearly efficiency over all SNR values). AR, ESPRIT and Unitary ESPRIT produce almost the same performances that are far from those given by the MLE procedures. Unitary ESPRIT with improved aperture significantly improves the MSE but does not perform as well as AMLE with gain difference about $10 \mathrm{~dB}$ over large SNR intervals.

\section{Conclusions}

This paper presented a low computational cost procedure to solve the conditional ML problem for direction finding of two closely spaced sources. It is based on an approximation of the concentrated likelihood function, assuming a small difference between the two DoA. The algorithm is easy to implement as relies on weighted Fourier transforms of the data. Its performance is very close to that of the exact MLE even for quite large DOA separation. It inherits all desirable properties of the ML estimator such as robustness to source correlation and single snapshot capabilities where few successful algorithms are known. To finish with and based on these results, the complementary problem of estimating the number of sources or analyzing the resolution threshold should then be investigated. This detection problem constitutes a natural perspective of this work.

\section{Appendix A. Taylor expansion of $P$}

In this appendix, we provide a sketch of proof for (11). Substitution of the Taylor expansions $\boldsymbol{D}=\sum \boldsymbol{D}_{k} \Delta_{f}^{k}$ and $c=\sum c_{k} \Delta_{f}^{k}$ into (8) immediately leads to

$\boldsymbol{P}=\frac{\boldsymbol{a} \boldsymbol{a}^{H}+\sum_{k, l} \Delta_{f}^{k+l}\left[\boldsymbol{D}_{k} \boldsymbol{a} \boldsymbol{a}^{H} \boldsymbol{D}_{l}^{H}-c_{k} \boldsymbol{a a}^{H} \boldsymbol{D}_{l}^{H}-c_{k}^{*} \boldsymbol{D}_{l} \boldsymbol{a} \boldsymbol{a}^{H}\right]}{1-\sum_{k, l} \Delta_{f}^{k+l} c_{k} c_{l}^{*}}$.

Now, using the facts that $\boldsymbol{D}_{0}=\boldsymbol{I}$ (since $f_{1}=f_{2}$ if $\Delta_{f}=0$ ) and that $c_{0}=1$, we have

$\boldsymbol{P}=-\frac{\sum_{n=1} \Delta_{f}^{n}\left(\sum_{k=0}^{n} \boldsymbol{D}_{k} \boldsymbol{a} \boldsymbol{a}^{H} \boldsymbol{D}_{n-k}^{H}-c_{k} \boldsymbol{a a}^{H} \boldsymbol{D}_{n-k}^{H}-c_{k}^{*} \boldsymbol{D}_{n-k} \boldsymbol{a a}^{H}\right)}{\sum_{n=1} \Delta_{f}^{n}\left(\sum_{k=0}^{n} c_{k} c_{n-k}^{*}\right)}$

which can be rewritten as

$\boldsymbol{P}=-\frac{\sum_{n=1} \Delta_{f}^{n} \boldsymbol{M}_{n}}{\sum_{n=1} \Delta_{f}^{n} d_{n}}$

with

$\boldsymbol{M}_{n}=\sum_{k=0}^{n} \boldsymbol{D}_{k} \boldsymbol{a} \boldsymbol{a}^{H} \boldsymbol{D}_{n-k}^{H}-c_{k} \boldsymbol{a a}^{H} \boldsymbol{D}_{n-k}^{H}-c_{k}^{*} \boldsymbol{D}_{n-k} \boldsymbol{a a}^{H}$

$d_{n}=\sum_{k=0}^{n} c_{k} c_{n-k}^{*}$

Observing that $c_{1}^{*}=-c_{1}$ (due to pure complex phase terms in the steering vector), it follows that $\boldsymbol{M}_{1}=\mathbf{0}$ and $d_{1}=0$. Therefore,

$$
\begin{aligned}
\boldsymbol{P} & =-\frac{\boldsymbol{M}_{2}+\boldsymbol{M}_{3} \Delta_{f}+\boldsymbol{M}_{4} \Delta_{f}^{2}+O\left(\Delta_{f}^{3}\right)}{d_{2}+d_{3} \Delta_{f}+d_{4} \Delta_{f}^{2}+O\left(\Delta_{f}^{3}\right)} \\
& \simeq-\frac{1}{d_{2}}\left(\boldsymbol{M}_{2}+\boldsymbol{M}_{3} \Delta_{f}+\left(\boldsymbol{M}_{4}-\frac{d_{4}}{d_{2}} \boldsymbol{M}_{2}\right) \Delta_{f}^{2}\right)+O\left(\Delta_{f}^{3}\right)
\end{aligned}
$$

where we used the fact that $d_{3}=0$. This proves that the second-order Taylor expansion of $\boldsymbol{P}$ is given by (11).

\section{References}

[1] H.L.V. Trees (Ed.), Optimum Array Processing, Wiley, New York, 2002.

[2] F.C. Schweppe, Sensor array data processing for multiple signal sources, IEEE Trans. Inf. Theory 14 (1968) 294-305.

[3] S.M. Kay, Fundamentals of Statistical Signal Processing: Estimation Theory, Prentice Hall, Englewood Cliffs, NJ, 1993.

[4] P. Stoica, A. Nehorai, Performance study of conditional and unconditional direction-of-arrival estimation, IEEE Trans. Acoust. Speech Signal Process. 38 (10) (1990).

[5] J. Capon, High-resolution frequency wavenumber spectrum analysis, Proc. IEEE 57 (1969) 1408-1418.

[6] G. Bienvenu, L. Kopp, Adaptivity to background noise spatial coherence for high resolution passive methods, in: Proceedings of the ICASSP, 1980, pp. 307-310.

[7] R.O. Schmidt, Multiple emitter location and signal parameter estimation, in: RADC Spectral Estimation Workshop, 1979, pp. 243-258.

[8] A.J. Barabell, Improving the resolution performance of eigenstructurebased direction-finding algorithms, in: Proceedings ICASSP, Boston, MA, 1983, pp. 336-339.

[9] B.D. Rao, K.V.S. Hari, Performance analysis of root-MUSIC, IEEE Trans. Acoust. Speech Signal Process. 37 (12) (1989) 1939-1949.

[10] R. Roy, T. Kailath, ESPRIT-estimation of signal parameters via rotational invariance techniques, IEEE Trans. Acoust. Speech Signal Process. 37 (7) (1989) 984-995.

[11] R. Kumaresan, D.W. Tufts, Estimating the angles of arrival of multiple plane waves, IEEE Trans. Aerospace Electron. Syst. 19 (1) (1983) 134-139.

[12] M. Pesavento, A.B. Gershman, M. Haardt, Unitary root-MUSIC with a real-valued eigendecomposition: a theoretical and experimental study, IEEE Trans. Signal Process. 48 (5) (2000) 1306-1314.

[13] M. Haardt, J. Nossek, Unitary ESPRIT: how to obtain increased estimation accuracy with a reduced computational burden, IEEE Trans. Signal Process 43 (5) (1995) 1232-1242.

[14] P. Stoica, A. Nehorai, MUSIC, maximum likelihood, and Cramér-Rao bound, IEEE Trans. Acoust. Speech Signal Process. 37 (5) (1989). 
[15] P. Stoica, K.C. Sharman, Maximum likelihood methods for directionof-arrival estimation, IEEE Trans. Acoust. Speech Signal Process. 38 (7) (1990).

[16] T.-J. Shan, M. Wax, T. Kailath, On spatial smoothing for direction-ofarrival estimation of coherent signals, IEEE Trans. Acoust. Speech Signal Process. 33 (4) (1985) 806-811.

[17] P. Häcker, B. Yang, Analytical investigation of two-object DOA estimation, in: Proceedings of the International ITG Workshop on Smart Antennas (WSA), Aachen, Germany, 2011.

[18] M. Schoor, High-resolution DOA estimation for automotive radar systems (Ph.D. thesis), Universität Stuttgart, 2010.

[19] P. Heidenreich, Antenna array processing: autocalibration and fast high-resolution methods for automotive radar (Ph.D. thesis), Technische Universität Darmstadt, 2012.

[20] I. Ziskind, M. Wax, Maximum likelihood localization of multiple sources by alternating projection, IEEE Trans. Acoust. Speech Signal Process. 36 (10) (1988).

[21] A.P. Dempster, N.M. Laird, D.B. Rubin, Maximum likelihood from incomplete data via the EM algorithm, J. R. Stat. Soc. 39 (1) (1977) $1-38$.

[22] A.J. Weiss, A.S. Willsky, B.C. Levy, Maximum likelihood array processing for the estimation of superimposed signals, Proc. IEEE 76 (2) (1988) 203-204.
[23] Y. Bresler, A. Makovski, Exact maximum likelihood parameter estimation of superimposed exponential signals in noise, IEEE Trans. Acoust. Speech Signal Process. 34 (5) (1986) 307-310.

[24] P. Stoica, K.C. Sharman, Novel eigenanalysis method for direction estimation, IEE Proc. Radar Sonar Navigation 137 (1) (1990).

[25] A. Swindlehurst, Alternative algorithm for maximum likelihood DOA estimation and detection, IEE Proc. Radar Sonar Navigation 141 (6) (1994).

[26] M. Kaveh, A. Barabell, The statistical performance of the MUSIC and the minimum-norm algorithms in resolving plane waves in noise IEEE Trans. Acoust. Speech Signal Process. 34 (2) (1986) 331-341.

[27] H.B. Lee, M.S. Wengrovitz, Resolution threshold of beamspace MUSIC for two closely spaced emitters, IEEE Trans. Acoust. Speech Signal Process. 38 (9) (1990) 1545-1559.

[28] C.D. Richmond, Capon algorithm mean-squared error threshold SNR prediction and probability of resolution, IEEE Trans. Signal Process. 53 (8) (2005) 2748-2764.

[29] U. Nickel, Applications of superresolution for radar: examples, problems and solutions, in: Proceedings of the Eurasip Eusipco, Marrakech, 2013.

[30] K.G.A. Thakre, M. Haardt, Single snapshot spatial smoothing with improved effective array aperture, IEEE Signal Process. Lett. 16 (6) (2009) 505-508. 